\title{
A Solution for Scalable Routing in Depth Divisions-Based DUSNs via Adding a Scalable Parameter to Control Depth Clusters: Creating an Energy Efficient and Low Delay NI-Independent Communication Protocol
}

\author{
Mohammad Reza Khosravi', Reza Salari', Habib Rostami2* \\ ${ }^{1}$ Department of Electrical Engineering, School of Engineering, Persian Gulf University, Bushehr Port, Iran \\ ${ }^{2}$ Department of Computer Engineering, School of Engineering, Persian Gulf University, Bushehr Port, Iran \\ Email: "habib@pgu.ac.ir
}

Received 4 April 2016; accepted 22 May 2016; published 25 May 2016

Copyright (C) 2016 by authors and Scientific Research Publishing Inc.

This work is licensed under the Creative Commons Attribution International License (CC BY).

http://creativecommons.org/licenses/by/4.0/

(c) (i) Open Access

\section{Abstract}

Due to effectiveness of network layer on general performance of networks, designing routing protocols is very important for lifetime and traffic efficiency in wireless sensor networks. So in this paper, we are going to represent an efficient and scalable version of depth-based routing (DBR) protocol that is limited by depth divisions-policy. In fact the new version is a network information independent routing protocol for acoustic communications. Proposed method by use of depth clustering is able to reduce consumed energy and end-to-end delay in dense underwater sensor networks (DUSNs) and this issue is proved by simulation.

\section{Keywords}

Network Information (NI), Depth-Based Routing (DBR), Dense Underwater Sensor Networks (DUSNs), Energy Efficient and Low Delay-DBR (EELD-DBR)

\section{Introduction}

Underwater network is a new communication area for research in recent years. In these networks, acoustic

${ }^{*}$ Corresponding author.

How to cite this paper: Khosravi, M.R., et al. (2016) A Solution for Scalable Routing in Depth Divisions-Based DUSNs via Adding a Scalable Parameter to Control Depth Clusters: Creating an Energy Efficient and Low Delay NI-Independent Communication Protocol. Journal of Computer and Communications, 4, 55-61. http://dx.doi.org/10.4236/jcc.2016.47008 
channel is used instead of electromagnetic channel as physical layer due to high electromagnetic wave [10] attenuations. Using this type of channel creates high delay and low bandwidth links in underwater communications, also inaccessibility to sun light and wired electrical resources in underwater space is a key limitation and creates energy constraints for the networks. Therefore, designing efficient communication protocols according to these challenging issues is essential. Designing routing protocols is very important due to effectiveness of network layer on general performance of the network. A key routing scheme to apply in underwater sensor networks is Depth-Based Routing (DBR) protocol [1]. This protocol has excellent suitability for dense network application. Based paper of DBR explicitly guarantees high data delivery ratio for dense networks. It is wellknown that dense networks have challenges in energy consumption and link delay [2]-[4], so we select DBR as basic routing protocol in this investigation.

In fact, the main aim is creating new version of DBR that has high delivery ratio, like DBR, and in addition reduces link delay and energy consumption of the networks. Our featured approach is using a scalable type of depth divisions-based policy [5] in a DBR-base network. The rest of paper is categorized as follow. 2nd section is allocated to review of basic concepts, 3rd section introduces proposed scheme and 4th section analyses proposed scheme. Final section is conclusion, too.

\section{An Overview on Basic Schemes}

DBR is a specific routing protocol among all underwater routing protocols because of the protocol does not have any need to foreign information. In fact, the protocol to perform routing is only depended to node's depth information and it is noticeable that using a simple depth finder is sufficient for obtaining routing information. Thus, DBR is called as a network information independent (NI-independent) protocol and sometimes as nongeographical routing policy. Network information means information achieved from a positioning system (such as GPS in VBF [8]) or exchanging control packets (or beacons). DBR for node's cooperation in routing has a condition, in fact uses an unequal condition to decide about cooperation of each receiving node of data packets. Figure 1 shows DBR details in a geometric diagram.

In this figure, node $(S)$ is sender. According to higher depth of node (n3), cooperation of this node as router in forwarding process is not useful because of this node is far from sink node (destination) and node (n3) should not perform packet broadcasting, Whereas nodes $(n 1)$ and $(n 2)$ are in suitable depth and should help routing process. In DBR protocol a depth threshold $\left(d_{t h}\right)$ has been defined to improve efficiency according to network density, for example node $(n 1)$ has better position than node $(n 2)$ to route the data packets, so with selection of " $d_{t h}<d_{s}-d_{n 2}$ ", only node ( $\left.n 1\right)$ will be allowed for cooperation as router (minimum of $d_{t h}$ is zero in theory). So after applying DBR as routing protocol, router nodes rebroadcast received packets and other nodes discard them. Discarding nodes are known as non-cooperative nodes, too. To cover other details of DBR, we must now that each node keeps a priority queue (Q1) and a packet history buffer (Q2). An item in $Q 2$ is a unique ID for each

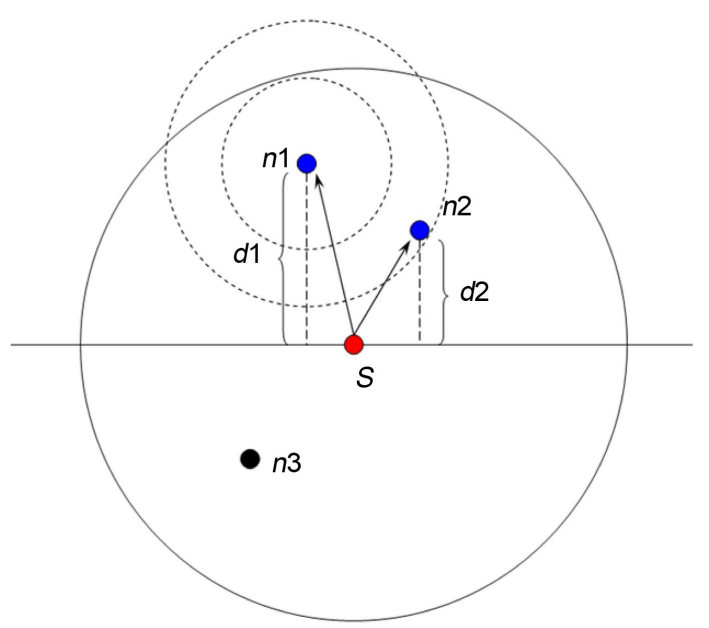

Figure 1. Showing the DBR routing protocol, DBR selects suitable nodes to cooperate in routing ${ }^{1}$.

${ }^{1}$ Figure 1 is extracted from [1]. 
packet, which is composed of Sender ID and Sequence Number of the Packet. When a node successfully transmits a packet, it inserts the unique ID of the packet into Q2. In other words, Q2 keeps a recent history of the packets the node has transmitted. Each item in $Q 1$ includes two particles: a packet and the scheduled sending time [1] for the packet. The priority of a case in $Q 1$ is explained by the scheduled sending time. More specifically, a case with earlier sending time has a higher priority. When a node receives a packet, instead of sending the packet immediately, it first holds the packet for a certain amount of time, called holding time. The scheduled sending time of a packet is computed based on the time while the packet is received and the known holding time for the packet [1]. However, note that DBR does not have the best link delay and energy consumption in high densities of the network, because of an independent control tool is not existent in DBR.

\section{Proposed Scheme: EELD-DBR}

Now, we are going to introduce our new improvement i.e. Energy Efficient and Low Delay-DBR (EELD-DBR), with this assumption that network is working in dense mode (density is depended on three factors: number of nodes, space volume and radiation range of router nodes [12]), we wish to create some depth routing clusters for improving routing performance. Note that the core of EELD-DBR yet is DBR. In the other word, in EELD-DBR we choose some suitable nodes to route data packets and name these nodes as cluster members. A region regarding to existence of some cluster members with same depth (approximately) is called as a cluster. Each routing cluster routes packets based on basic routing protocol i.e. DBR. In here, we don't consider cluster heads, in fact all nodes are separated in two categories; first category is non-cooperative nodes (this definition is different from definitions in DBR, seen previously) that are out of routing clusters. Because of creation of routing clusters based on depth, we call the clusters as depth routing clusters or depth clusters. The rest of nodes (second category) are included in routing clusters. Only second group of nodes can act as router. In our previous works [5] [6] that have been published recently, this issue was proved that independent zoning from basic routing protocol in underwater space is effective on energy consumption, but these works have weaknesses. In [5], the depth divisions-based routing policy for routing enhancement of dense networks is represented but this energy efficiency is not proper for scalable networks, also both researches are not attention to delay issue. In fact, we are going to integrate all of these enhancements to create a powerful enhancement that has energy efficiency, delay reduction and also against previous researches [5] [6], introduces an adaptive approach based on the depth divisions-based routing policy discussed in [5] and also based on DBR basic protocol to create a new NI-independent routing protocol. Algorithm 1 and also Figure 2 explicitly illustrate depth clustering in EELD-DBR. In this figure, assume that underwater space is defined as $1000 \times 1000 \times 2000 \mathrm{~m}^{3}$ (height $(H)$ is equal to 2000 meters) and

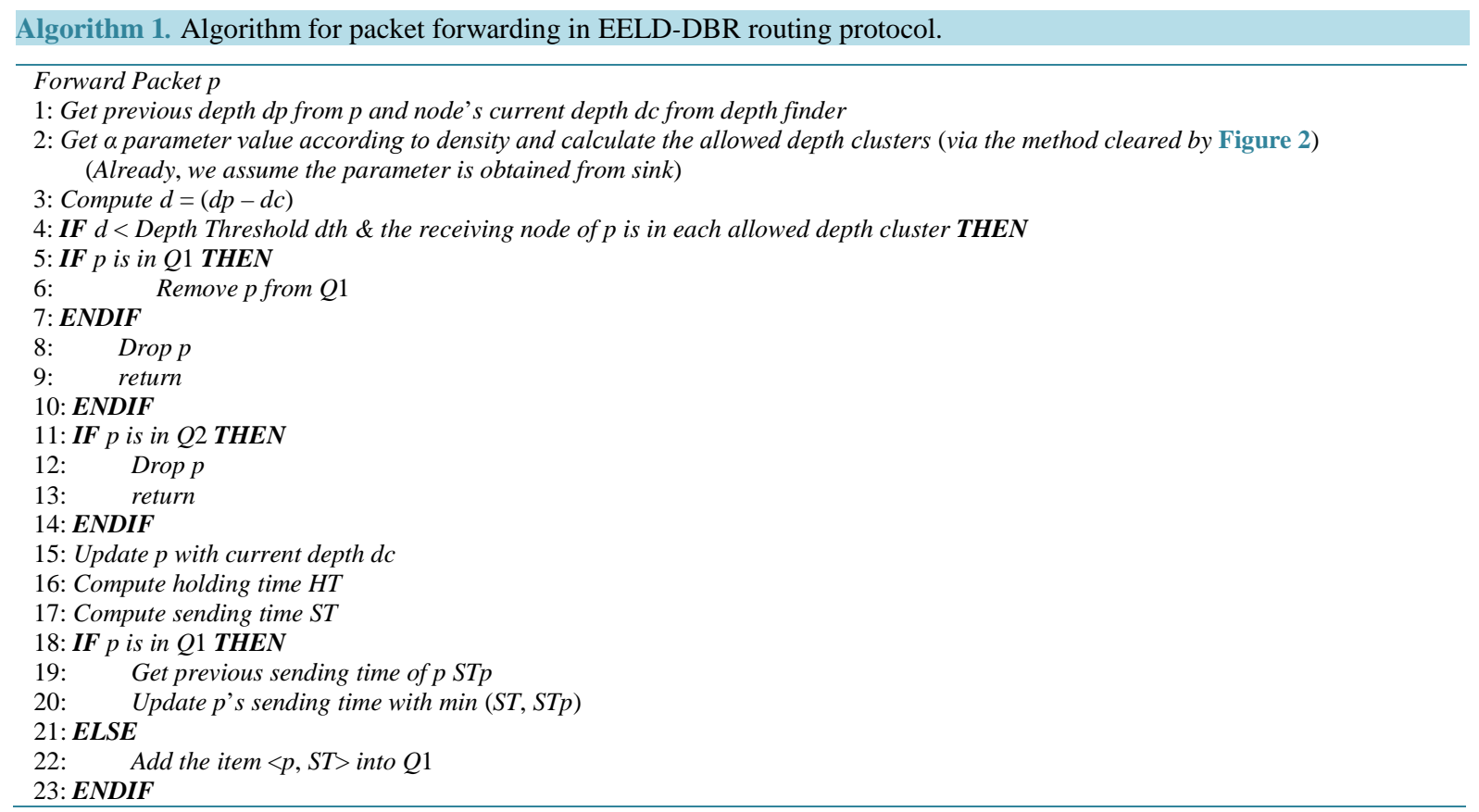




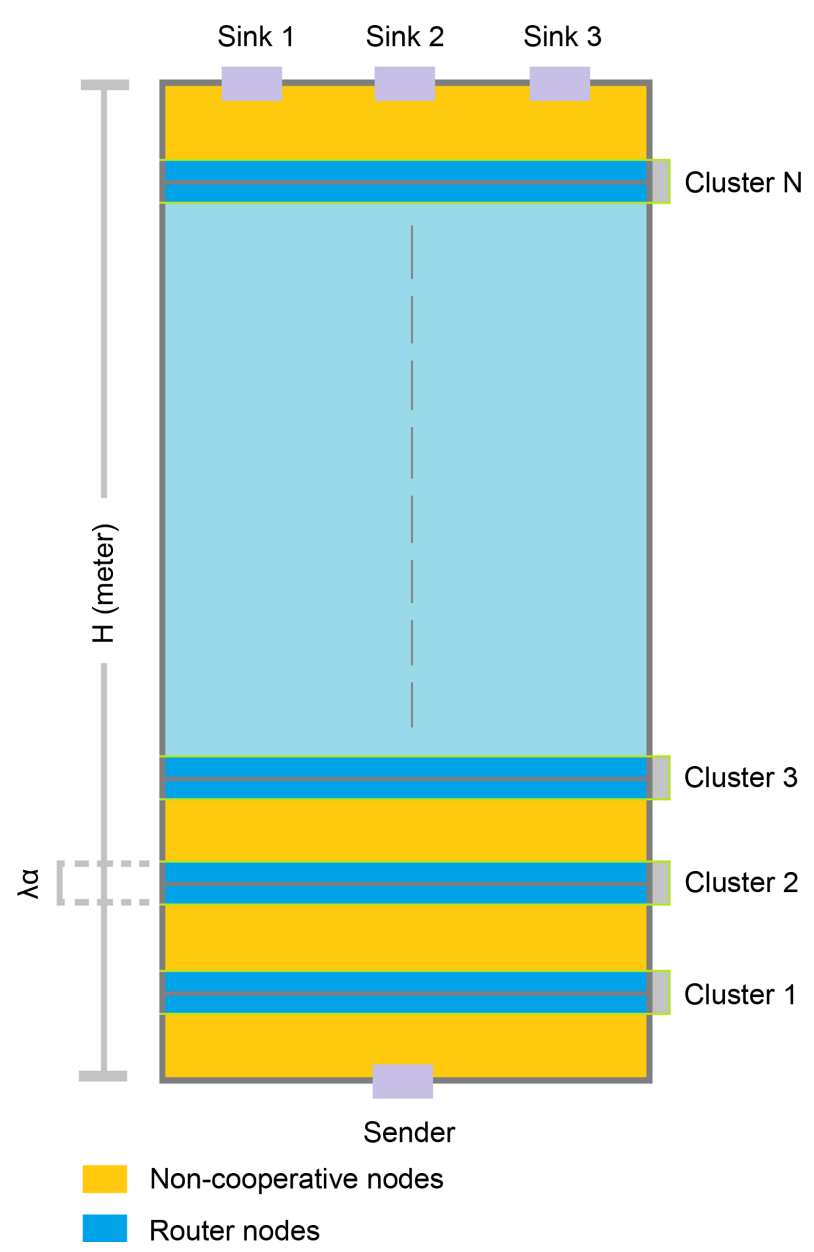

Figure 2. Depth clustering, this figure shows applying an adaptive space zoning over a DBR-based dense network.

acoustic range of each node's communication modem $(\lambda)$ is considered 100 meters, single sender node is in the bottom of 3D space (depth is equal to 2000 meters) and several sink nodes are at the top of space (depth is equal to 0 ). So, we can create 19 clusters from Cluster 1 to Cluster 19 (Cluster $N$ ) that central depth of each cluster has distance of $100 \mathrm{~m}$ (i.e. equal to $\lambda$ ) from central depth of its lower and higher neighbor clusters. Width of each cluster will be $\lambda \alpha$ meter(s) that $\alpha$ is adaptation parameter for scalability, for example if $\alpha$ is 0.4 , in this example width of each cluster will be 40 meters. So, it is observable that number of clusters $(N)$ is depended to $H$ and $\lambda$. For simplicity, we assume that $H$ is dividable on $\lambda$, thus Equation (1) shows relation among $N, \lambda$ and $H$ :

$$
N=\frac{H}{\lambda}-1
$$

Parameter $\alpha$ is defined for creating adaptation and scalability. In very dense mode, we can select a small value for $\alpha$ and in sparse mode, we can vary it to its maximum value, i.e. 1 , so $\alpha$ is between 0 and 1 . It is noticeable that with assumption an uniform distribution of nodes in 3D underwater space, maximum number of router nodes according to Equation (2) is limited to $M \alpha(1-\lambda / H)$. In here, $M$ shows number of all sensor nodes that can be as router.

$$
\begin{aligned}
\text { Routers Number }_{\max } & =M \times \frac{N \lambda \alpha}{H}=\frac{M \lambda \alpha}{H}\left(\frac{H}{\lambda}-1\right) \\
& =\frac{M H \alpha-M \lambda \alpha}{H}=M \alpha\left(1-\frac{\lambda}{H}\right)
\end{aligned}
$$


In addition of energy saving, existing big gaps in depth clustering, helps to reduce delay of links, because of packet trajectory from sender to sink is shorter than ordinary DBR. Next, we will see effectiveness of proposed scheme versus ordinary scheme.

\section{Numerical Results Based on Simulation}

Delivery ratio in all simulations is held on at least 90\%. For evaluation of proposed scheme, underwater space and acoustic range are assumed as the example in previous part. NS-2 software is used for simulation. MAC protocol is IEEE 802.11, transport layer protocol is UDP [5] and application layer protocol is CBR [5]. Number of nodes (density component) is varied between 1000 to 3000 nodes and mobility of nodes is selected $1 \mathrm{~m} / \mathrm{s}$ and $5 \mathrm{~m} / \mathrm{s}$ for simulations (in each simulation, all nodes have equivalent velocity). $\alpha$ is equal to 0.4 for EELD-DBR and $d_{t h}$ is zero in all simulations and for all nodes. Figures 3-6 show consumed energy and total delay comparisons

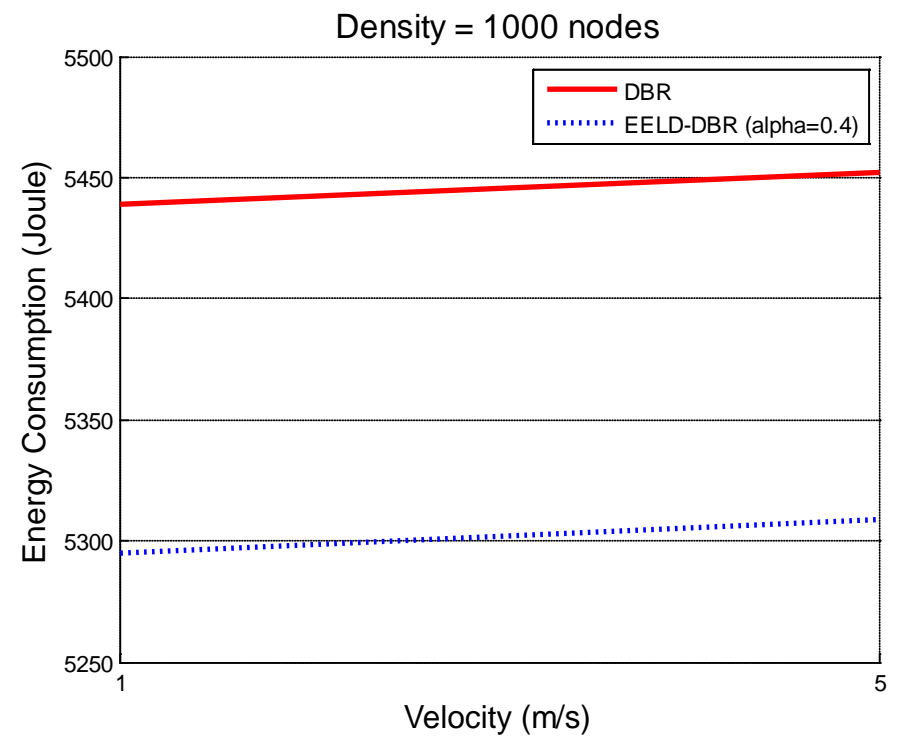

Figure 3. Consumed energy between DBR and EELD-DBR, consumed energy results are in joule unit, network density is fixed and equal to 1000 nodes and also velocity (mobility of all nodes) is selected between 1 and $5 \mathrm{~m} / \mathrm{s}$.

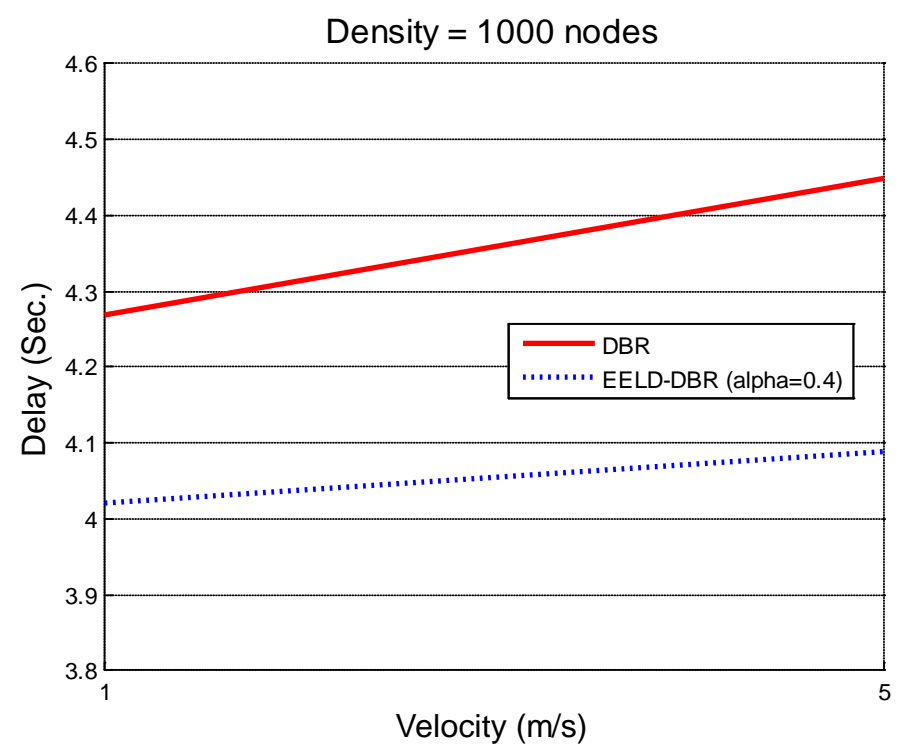

Figure 4. Delay comparison between DBR and EELD-DBR, delay results are in second unit, network density is fixed and equal to 1000 nodes and also velocity (mobility of all nodes) is selected between 1 and $5 \mathrm{~m} / \mathrm{s}$. 


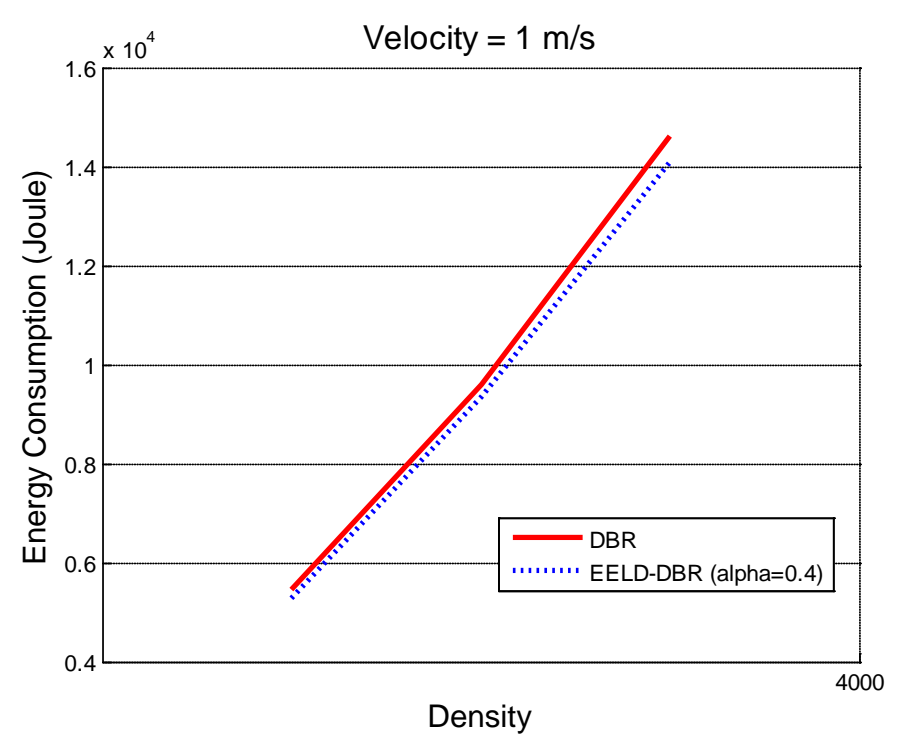

Figure 5. Consumed energy between DBR and EELD-DBR, consumed energy results are in joule unit, network density has variations from 1000 to 3000 nodes and also velocity (mobility of all nodes) is $1 \mathrm{~m} / \mathrm{s}$ (fixed).

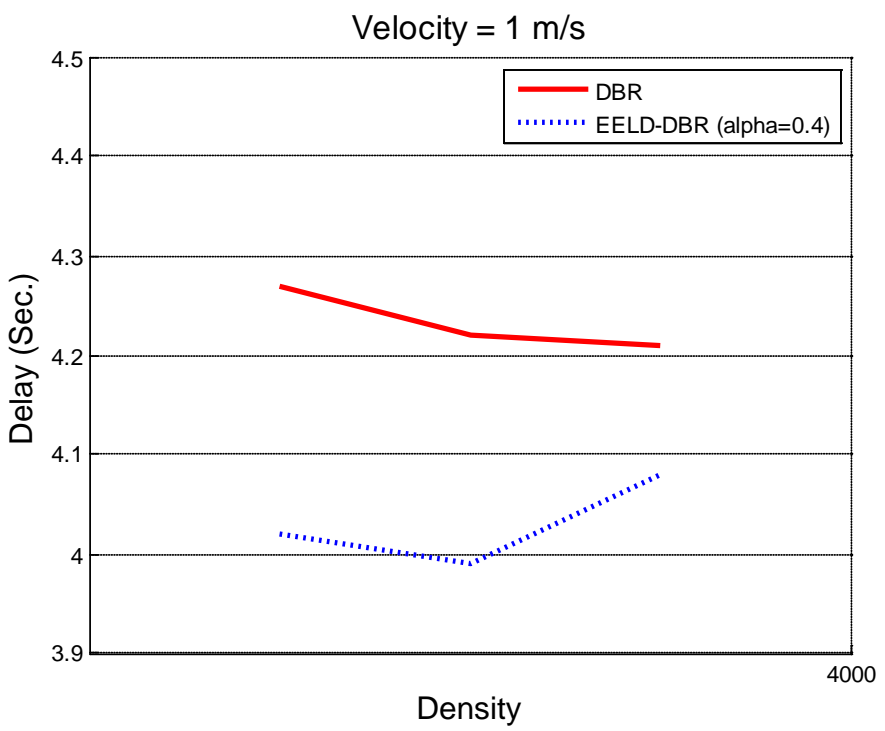

Figure 6. Delay comparison between DBR and EELD-DBR, delay results are in second unit, network density has variations from 1000 to 3000 nodes and also velocity (mobility of all nodes) is $1 \mathrm{~m} / \mathrm{s}$ (fixed). This figure clearly illutrates lower delay of EELD-DBR than DBR.

between DBR and EELD-DBR during 200 seconds (as simulation time). This figures show lower energy consumption and link delay of EELD-DBR in comparison with DBR.

Figure 3 and Figure 4 are in constant density of 1000-node with various speeds and evaluate the schemes in terms of consumed energy and link delay, respectively. Figure 5 and Figure 6 are in constant mobility of $1 \mathrm{~m} / \mathrm{s}$ with various densities and evaluate the schemes in respect with consumed energy and link delay, respectively. So, proposed method by use of depth clustering is able to reduce consumed energy and end-to-end delay in dense underwater sensor networks (DUSNs). All simulations are accurate and performed in a space of discrete-event with finite experimental points to figure curves.

\section{Conclusion and Future Works}

We introduced our new improvement over DBR protocol, i.e. Energy Efficient and Low Delay-DBR (EELD- 
DBR) for underwater wireless sensor networks, with this assumption that network is working in dense mode. This enhancement is applicable in all dense networks with different routing protocols. For future work, we can create an adaptive version of EELD-DBR by use of component adaptation concept [7], i.e. a protocol that in it, each router node can select a specific scalable parameter $(\alpha)$ for itself; this value can be different from other each node's scalable parameter. This paper is directly an enhancement on our previous papers [5] [6] [9] [11] that are published in 2015 and in fact DBR as basic routing scheme can be changed to any other protocol. Of course, using DBR is necessary, if we are going to create a NI-independent routing protocol.

\section{References}

[1] Yan, H., Shi, Z. and Cui, J. (2008) DBR: Depth-Based Routing for Underwater Sensor Networks. Proceedings of the 7th international IFIP-TC6 Networking Conference on Ad-Hoc and Sensor Networks, 4982, 72-86. http://dx.doi.org/10.1007/978-3-540-79549-0_7

[2] Ayaz, M., Baig, I. and Abdullah, A. (2011) A Survey on Routing Techniques in Underwater Wireless Sensor Networks. Journal of Network and Computer Applications, 34, 1908-1927. http://dx.doi.org/10.1016/j.jnca.2011.06.009

[3] Xie, P. (2010) Efficient Vector-Based Forwarding for Underwater Sensor Networks. EURASIP Journal on Wireless Communications and Networking, 2010, Article ID: 195910. http://dx.doi.org/10.1155/2010/195910

[4] Han, G. and Bao, N. (2015) Routing Protocols in Underwater Acoustic Sensor Networks: A Quantitative Comparison. International Journal of Distributed Sensor Networks, 2015, Article ID: 858593. http://dx.doi.org/10.1155/2015/858593

[5] Khosravi, M.R., Basri, H. and Rostami, H. (2015) Improvement of Energy Consumption in Dense Underwater Sensor Networks. 2nd International Congress of Electrical Engineering, Computer Science and Information Technology (IT2015), SBU, Tehran, 9, 1-9. http://dl.pgu.ac.ir/handle/Hannan/17956

[6] Khosravi, M.R., Basri, H., Khosravi, A. and Rostami, H. (2015) Energy Efficient Spherical Divisions for VBF-Based Routing in Dense UWSNs. IEEE2015-The Second International Conference on Knowledge-Based Engineering and Innovation (KBEI), IUST, Tehran, 961-965. http://dx.doi.org/10.1109/KBEI.2015.7436174

[7] Yu, H., Yao, N. and Liu, J. (2014) An Adaptive Routing Protocol in Underwater Sparse Acoustic Sensor Networks. Ad-Hoc Networks, 34, 121-143. http://dx.doi.org/10.1016/j.adhoc.2014.09.016

[8] Khosravi, M.R., Basri, H. and Rostami, H. (2015) Routing with Using Vector-Based Forwarding in Underwater Wireless Sensor Networks. 2nd International Congress of Electrical Engineering, Computer Science and Information Technology (IT2015), SBU, Tehran, Iran, 9, 10-20. http://dl.pgu.ac.ir/handle/Hannan/17957

[9] Khosravi, M.R. (2015) Routing Analysis and Evaluation for Underwater Acoustic Sensor Networks. M.Sc. Thesis in Electrical Engineering, Persian Gulf University, Bushehr.

[10] Wu, B., Yin, H., Liu, A., Liu, C. and Wang, J. (2016) Design and Implementation of Hybrid Light and Microwave Switches Based on Wavelength Selective Switch for Future Satellite Networks. Journal of Computer and Communications, 4, 14-19. http://dx.doi.org/10.4236/jcc.2016.43003

[11] Khosravi, M.R., Basri, H. and Rostami, H. (2016) Energy Efficient Random Cooperations for VBF-Based Routing in Dense UWSNs. International Conference on New Research Achievements in Electrical and Computer Engineering (ICNRAECE 2016), AUT, Tehran.

[12] Ansari, M.I.H., Singh, S.P. and Doja, M.N. (2016) Effect of Transmission Range on Ad Hoc on Demand Distance Vector Routing Protocol. Journal of Computer and Communications, 4, 34-46. http://dx.doi.org/10.4236/jcc.2016.42005 